\title{
Universal Large Deviations for the Tagged Particle in Single File Motion
}

\author{
Chaitra Hegde, ${ }^{1}$ Sanjib Sabhapandit, ${ }^{1}$ and Abhishek Dhar ${ }^{2}$ \\ ${ }^{1}$ Raman Research Institute, Bangalore - 560080, India \\ ${ }^{2}$ International centre for theoretical sciences, TIFR, Bangalore - 560012, India
}

(Dated: March 26, 2021)

\begin{abstract}
We consider a gas of point particles moving in a one-dimensional channel with a hard-core inter-particle interaction that prevents particle crossings - this is called single-file motion. Starting from equilibrium initial conditions we observe the motion of a tagged particle. It is well known that if the individual particle dynamics is diffusive, then the tagged particle motion is sub-diffusive, while for ballistic particle dynamics, the tagged particle motion is diffusive. Here we compute exactly the large deviation function for the tagged particle displacement and show that this is universal, independent of the individual dynamics.
\end{abstract}

PACS numbers: 05.40.-a, 83.50.Ha, 87.16.dp, 05.60.Cd

The motion of particles in narrow channels where the particles cannot overtake each other is referred to as single-file motion [see Fig. (1)]. This concept was introduced by Hodgkin and Keynes [1] to describe ion transport in biological channels. The motion of a tagged particle in such a single-file system has been of great interest since the classic papers by Jepsen [2] and Harris [3]. These papers showed that, in a gas of hard rods evolving with Hamiltonian dynamics, a tagged particle moves diffusively [2] with the mean square displacement (MSD) growing linearly with time $t$, whereas for a gas of Brownian particles, the tagged particle shows subdiffusion [3] with the MSD growing as $\sqrt{t}$. There has been a revival of interest in tagged particle diffusion as several experiments are now able to observe this in single-file systems in both colloidal and atomic single-file systems [4-9], and some of the theoretical predictions have been verified.

There have been a number of studies to understand tagged particle motion in systems with deterministic as well as stochastic dynamics [10-27]. Attempts have been made to obtain the full probability density function (PDF) for the tagged particle displacement. The $N$-particle propagator has been obtained using the "reflection principle" [16] and Bethe Ansatz [18], and from this the tagged particle distribution has been obtained by integrating out all other particles. However, the resulting form of the distribution is complicated and not very illuminating. An approximate scheme relying on Jepsen's mapping to non-interacting particles has been used in [19, 22]. A recent work [28] has used macroscopic fluctuation theory [29] to compute the cumulant generating function (CGF) corresponding to the tagged particle PDF.

In this Letter, we show that it is possible to exactly compute the large time asymptotic form of the PDF of tagged particle displacement. Our method is applicable to deterministic as well as stochastic systems that are initially in equilibrium. This leads to a universal form for the PDF. We consider a collection of hard-point identical particles distributed with an uniform density $\rho$ on the one dimensional line from $-\infty$ to $\infty$. Each particle moves independently using the same dynamics, except that the hard-core repulsion prevents crossing of particles. We consider a single-particle propagator of the

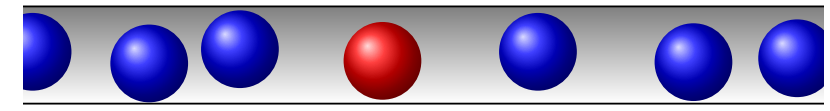

FIG. 1: (Color online) A schematic diagram of single-file motion of particles in a narrow channel where they cannot pass each other. We study the motion of a single tagged particle (say the red colored one).

general form

$$
G(y, t \mid x, 0)=\frac{1}{\sigma_{t}} f\left(\frac{y-x}{\sigma_{t}}\right),
$$

where $f(-w)=f(w) \geq 0, \int_{-\infty}^{\infty} f(w) d w=1$, and \langle|$y-$ $x|\rangle / \sigma_{t}=\int_{-\infty}^{\infty}|w| f(w) d w=\Delta$ is finite. Using a mapping to the non-interacting gas picture, we show that the PDF of the displacement $X_{t}$, of the tagged particle, has the large deviation form

$$
P_{\operatorname{tag}}\left(X_{t}, t \mid 0,0\right) \sim e^{-\rho \sigma_{t} I\left(X_{t} / \sigma_{t}\right)},
$$

where the large deviation function (LDF) is given exactly by

$$
\begin{aligned}
I(z) & =2 Q(z)-\left[4 Q^{2}(z)-z^{2}\right]^{1 / 2}, \\
\text { with } \quad Q(z) & =z \int_{0}^{z} f(w) d w+\int_{z}^{\infty} w f(w) d w .
\end{aligned}
$$

We also compute the leading order correction exactly [see Eqs. (19) and [20].

We first outline the strategy used in the calculation. Initially, we consider $2 N+1$ particles, independently and uniformly distributed in the interval $[-L, L]$. In the computation, we assume both $N$ and $L$ to be large and keep only the dominant term. Finally, we take the limit $N \rightarrow \infty, L \rightarrow \infty$ while keeping $N / L=\rho$ fixed. Since during a collision each particle acts as a reflecting hard wall for the other and the particles are identical, one can effectively treat the system of the interacting hard-point particles as non-interacting by exchanging the identities of the particles emerging from collisions. In the non-interacting picture, each particle executes an independent 
motion and the particles pass through each other when they 'collide'. The position of each particle at time $t$ is given independently by the propagator in Eq. (1). In many physical problems, the propagator is Gaussian, i.e., $f(x)=e^{-x^{2} / 2} / \sqrt{2 \pi}$, where $\sigma_{t}$ is simply the standard deviation. For example, for Hamiltonian dynamics with initial velocities chosen independently from Gaussian distribution with zero mean and variance $\bar{v}^{2}$ we have $\sigma_{t}=\bar{v} t$. On the other hand, for Brownian particles, $\sigma_{t}=\sqrt{2 D t}$, where $D$ is the diffusion coefficient. For fractional Brownian motion, $\sigma_{t} \propto t^{H}$, where $H$ is the Hurst exponent. However, our analysis is valid for a general propagator. Note that the dependence on time only appears through the characteristic displacement $\sigma_{t}$ in time $t$.

The joint probability density of the middle tagged particle being at $x$ at time $t=0$, and at $y$ at time $t$, can be expressed in terms of properties of the non-interacting particles. In the noninteracting picture, there are two possibilities: (i) the middle particle at time 0 is still the middle particle at time $t$, (ii) a second particle has become the middle particle at time $t$. We need to sum over these two processes.

To compute the contribution from process (i) we pick one of the non-interacting particles at random with a density $\rho$, multiply by the propagator [Eq. [1]] that it goes from $(x, 0)$ to $(y, t)$, and then multiply by the probability that it is the middle particle at both $t=0$ and $t$. Thus one obtains:

$$
P_{(1)}(x, 0 ; y, t)=\rho G(y, t \mid x, 0) F_{1 N}(x, y, t),
$$

where $F_{1 N}(x, y, t)$ is the probability that there are an equal number of particles to the left and right of $x$ and $y$ at $t=0$ and $t$ respectively.

To compute the contribution from process (ii), we first pick two particles at random at time $t=0$, and multiply by the propagators that they go from $(x, 0)$ to $(\tilde{y}, t)$ and $(\tilde{x}, 0)$ to $(y, t)$ respectively. We then multiply by the probability there are an equal number of particles on both sides of $x$ and $y$ at $t=0$ and $t$ respectively. Finally, integrating with respect to $\tilde{x}, \tilde{y}$, we get

$$
\begin{aligned}
P_{(2)}(x, 0 ; y, t) & =\rho^{2} \int_{-\infty}^{\infty} d \tilde{x} \int_{-\infty}^{\infty} d \tilde{y} \\
& \times G(\tilde{y}, t \mid x, 0) G(y, t \mid \tilde{x}, 0) F_{2 N}(x, y, \tilde{x}, \tilde{y}, t),
\end{aligned}
$$

where $F_{2 N}(x, y, \tilde{x}, \tilde{y}, t)$ is the probability that there are an equal number of particles on both sides of $x$ and $y$ at $t=0$ and $t$ respectively, given that there is a particle at $\tilde{x}$ at time $t=0$, and a particle at $\tilde{y}$ at time $t$. The joint PDF of the tagged particle is exactly given by

$$
P(x, 0 ; y, t)=P_{(1)}(x, 0 ; y, t)+P_{(2)}(x, 0 ; y, t) .
$$

To proceed further, we need the expressions for $F_{1 N}$ and $F_{2 N}$. Let $p_{-+}(x, y, t)$ be the probability that a particle is to the left of $x$ at $t=0$ and to the right of $y$ at time $t$. Similarly, we define the other three complementary probabilities. Clearly,

$$
\begin{aligned}
& p_{-+}(x, y, t)=(2 L)^{-1} \int_{-L}^{x} d x^{\prime} \int_{y}^{\infty} d y^{\prime} G\left(y^{\prime}, t \mid x^{\prime}, 0\right), \\
& p_{+-}(x, y, t)=(2 L)^{-1} \int_{x}^{L} d x^{\prime} \int_{-\infty}^{y} d y^{\prime} G\left(y^{\prime}, t \mid x^{\prime}, 0\right), \\
& p_{--}(x, y, t)=(2 L)^{-1} \int_{-L}^{x} d x^{\prime} \int_{-\infty}^{y} d y^{\prime} G\left(y^{\prime}, t \mid x^{\prime}, 0\right), \\
& p_{++}(x, y, t)=(2 L)^{-1} \int_{x}^{L} d x^{\prime} \int_{y}^{\infty} d y^{\prime} G\left(y^{\prime}, t \mid x^{\prime}, 0\right),
\end{aligned}
$$

and $p_{++}+p_{+-}+p_{-+}+p_{--}=1$. In terms of these probabilities, $F_{1 N}$ can be expressed as [30],

$$
F_{1 N}(x, y, t)=\int_{-\pi}^{\pi} \frac{d \phi}{2 \pi} \int_{-\pi}^{\pi} \frac{d \theta}{2 \pi}[H(x, y, \theta, \phi, t)]^{2 N},
$$

where

$$
\begin{aligned}
H(x, y, \theta, \phi, t) & =p_{++}(x, y, t) e^{i \phi}+p_{--}(x, y, t) e^{-i \phi} \\
& +p_{+-}(x, y, t) e^{i \theta}+p_{-+}(x, y, t) e^{-i \theta} .
\end{aligned}
$$

The angular integrals enforce the condition that the total number of particles crossing the middle particle from left-to-right is the same as the total number from right-to-left. This can be seen by explicitly performing the multinomial expansion above and computing the angular integrals. Using the fact that $2 N$ is even and the integrand is unchanged if both $\theta$ and $\phi$ are shifted by $\pi$ we can write $F_{1 N}$ in the form

$$
F_{1 N}(x, y, t)=\int_{-\pi / 2}^{\pi / 2} \frac{d \phi}{\pi} \int_{-\pi}^{\pi} \frac{d \theta}{2 \pi}[H(x, y, \theta, \phi, t)]^{2 N} .
$$

Similar argument can be used to compute $F_{2 N}$. However, in this case, one has to keep track of the order of the positions $(x, \tilde{x})$ and $(y, \tilde{y})$. One finds [30]

$$
\begin{aligned}
F_{2 N}(x, y, \tilde{x}, \tilde{y}, t)=\int_{-\pi / 2}^{\pi / 2} \frac{d \phi}{\pi} \int_{-\pi}^{\pi} & \frac{d \theta}{2 \pi}[H(x, y, \theta, \phi, t)]^{2 N-1} \\
& \times \psi(\theta, \phi \mid x, y, \tilde{x}, \tilde{y}),
\end{aligned}
$$

where the extra phase factor is given piece-wise by $\psi=e^{-i \phi}$, $e^{i \phi}, e^{-i \theta}$, and $e^{i \theta}$ for the situations (a) $\tilde{x}<x$ and $\tilde{y}<y$, (b) $\tilde{x}>x$ and $\tilde{y}>y$, (c) $\tilde{x}<x$ and $\tilde{y}>y$, and (d) $\tilde{x}>x$ and $\tilde{y}<y$ respectively.

Now, substituting the above form of $F_{2 N}$ in Eq. (5), and performing the integration over $\tilde{x}$ and $\tilde{y}$, while using the property $G(y, t \mid x, 0)=G(y-x, t \mid 0,0)$, we get

$$
\begin{gathered}
P_{(2)}(x, 0 ; y, t)=\rho^{2} \int_{-\pi / 2}^{\pi / 2} \frac{d \phi}{\pi} \int_{-\pi}^{\pi} \frac{d \theta}{2 \pi}[H(x, y, \theta, \phi, t)]^{2 N-1} \\
\times\left[2 A_{1}(z) A_{2}(z) \cos \phi+A_{1}^{2}(z) e^{-i \theta}+A_{2}^{2}(z) e^{i \theta}\right]
\end{gathered}
$$

where $z=(y-x) / \sigma_{t}$ and the functions $A_{1,2}(z)$ are given by

$$
A_{1}(z)=\int_{\sigma_{t} z}^{\infty} G(x, t \mid 0,0) d x=\int_{z}^{\infty} f(w) d w,
$$$$
\text { and } A_{2}(z)=1-A_{1}(z) \text {. }
$$ 
Now we explicitly compute the expressions for $p_{ \pm \pm}$using Eq. (1). Keeping only the dominant terms up to $O(1 / L)$, which survive in the limit $N \rightarrow \infty, L \rightarrow \infty$ while keeping $N / L=\rho$ fixed, we get

$$
\begin{aligned}
& p_{-+}=\frac{\sigma_{t}}{2 L}\left[-\frac{z}{2}+Q(z)\right]+\cdots \\
& p_{+-}=\frac{\sigma_{t}}{2 L}\left[\frac{z}{2}+Q(z)\right]+\cdots \\
& p_{--}=\frac{1}{2}+\frac{\sigma_{t}}{2 L}[\bar{z}-Q(z)]+\cdots \\
& p_{++}=\frac{1}{2}+\frac{\sigma_{t}}{2 L}\left[-\frac{\bar{z}}{2}-Q(z)\right]+\cdots,
\end{aligned}
$$

where $z=(y-x) / \sigma_{t}, \bar{z}=(y+x) / \sigma_{t}$, and the function $Q(z)$ is given by Eq. 3b.

To compute $H^{2 N}$ for large $N$, it is useful to express $H$ in the form

$$
\begin{aligned}
H & =1-(1-\cos \phi)\left(p_{++}+p_{--}\right)+i \sin \phi\left(p_{++}-p_{--}\right) \\
& -(1-\cos \theta)\left(p_{+-}+p_{-+}\right)+i \sin \theta\left(p_{+-}-p_{-+}\right) .
\end{aligned}
$$

Now, substituting $p_{ \pm \pm}$in the above expression of $H$, for large $N$, keeping only the most dominant terms, one finds

$$
H^{2 N}=e^{-2 N(1-\cos \phi)} e^{-i \rho \sigma_{t} \bar{z} \sin \phi} e^{-2 \rho \sigma_{t} Q(z)(1-\cos \theta)} e^{i \rho \sigma_{t} z \sin \theta} .
$$

Thus we have explicitly obtained $P_{(1)}, P_{(2)}$ and hence $P(x, 0 ; y, t)$ defined in Eq. 66. Using this we can finally write down the propagator for the displacement $X_{t}=y-x$ of the tagged particle as $P_{\operatorname{tag}}\left(X_{t}, t \mid 0,0\right)=\iint \delta\left(X_{t}-[y-\right.$ $x]) P(x, 0 ; y, t) d x d y$. Now making a change of variables from $x, y$ to $z, \bar{z}$, we get

$$
\begin{aligned}
& P_{\mathrm{tag}}\left(X_{t}=\sigma_{t} z, t \mid 0,0\right)= \\
& \lim _{N \rightarrow \infty} \int_{-\infty}^{\infty} \frac{d \bar{z}}{2} \int_{-\pi / 2}^{\pi / 2} \frac{d \phi}{\pi} \int_{-\pi}^{\pi} \frac{d \theta}{2 \pi} \rho B(z, \theta, \phi) \\
& \times e^{-2 N(1-\cos \phi)} e^{-i \rho \sigma_{t} \bar{z} \sin \phi} e^{-2 \rho \sigma_{t} Q(z)(1-\cos \theta)} e^{i \rho \sigma_{t} z \sin \theta},
\end{aligned}
$$

where $B(z, \theta, \phi)=f(z)+\rho \sigma_{t}\left[2 A_{1}(z) A_{2}(z) \cos \phi+\right.$ $\left.A_{1}^{2}(z) e^{-i \theta}+A_{2}^{2}(z) e^{i \theta}\right]$. For large $N$, the major contribution of the integral over $\phi$ comes from the region around $\phi=0$. Therefore, the $\phi$ integral can be performed by expanding around $\phi=0$ to make it a Gaussian integral (while extending the limits to $\pm \infty$ ). Subsequently, one can also perform the Gaussian integral over $\bar{z}$. This leads to the exact expression,

$$
\begin{array}{r}
P_{\operatorname{tag}}\left(X_{t}=\sigma_{t} z, t \mid 0,0\right)=\frac{1}{\sigma_{t}} \int_{-\pi}^{\pi} \frac{d \theta}{2 \pi} B(z, \theta) \\
\times e^{-\rho \sigma_{t}[2 Q(z)(1-\cos \theta)-i z \sin \theta],}
\end{array}
$$

where

$$
\begin{aligned}
& B(z, \theta) \equiv B(z, \theta, 0) \\
& =f(z)+\rho \sigma_{t}\left[2 A_{1}(z) A_{2}(z)+A_{1}^{2}(z) e^{-i \theta}+A_{2}^{2}(z) e^{i \theta}\right] .
\end{aligned}
$$

Since $\sigma_{t}$ is an increasing function of time, the integral over $\theta$ can be evaluated for large $t$, using the saddle point approximation. This gives the large deviation form given by Eq. (2) with the large deviation function given by

$$
I(z)=2 Q(z)\left(1-\cos \theta^{*}\right)-i z \sin \theta^{*}, \text { with } \tan \theta^{*}=\frac{i z}{2 Q(z)} .
$$

Eliminating $\theta^{*}$ yields the form given by Eq. 3a. The full asymptotic form of the propagator of the tagged particle displacement, obtained from the saddle point approximation is

$$
P_{\mathrm{tag}}\left(X_{t}=\sigma_{t} z, t \mid 0,0\right) \approx \frac{1}{\sigma_{t}} \frac{\sqrt{\rho \sigma_{t}}}{\sqrt{2 \pi g_{2}(z)}} g_{1}(z) e^{-\rho \sigma_{t} I(z)},
$$

where $g_{2}(z)=\left[4 Q^{2}(z)-z^{2}\right]^{1 / 2}$ has come from performing the Gaussian integral around the saddle-point $\theta^{*}$ and

$$
\begin{aligned}
& g_{1}(z)=\left(\rho \sigma_{t}\right)^{-1} B\left(z, \theta^{*}\right) \\
& =2 A_{1}(z) A_{2}(z)+A_{1}^{2}(z) \frac{\sqrt{2 Q(z)+z}}{\sqrt{2 Q(z)-z}}+A_{2}^{2}(z) \frac{\sqrt{2 Q(z)-z}}{\sqrt{2 Q(z)+z}} \\
& \quad+O\left(\left[\rho \sigma_{t}\right]^{-1}\right) .
\end{aligned}
$$

Note that the process (i) where, in the non-interacting picture, the same particle happens to be the middle particle at both the initial and final times, does not contribute at this order, but to $O\left(\left[\rho \sigma_{t}\right]^{-1}\right)$ in the expression of $g_{1}(z)$. In fact, one can systematically obtain the corrections to the above expression of $g_{1}(z)$, order by order. By keeping terms beyond the secondorder in the expansion of the argument of the exponential function around the saddle-point $\theta^{*}$ in Eq. (17), subsequently expanding the exponentials of the higher order terms in power series, and also expanding $B(z, \theta)$ around $\theta^{*}$ in power series, the resulting integrals in Eq. (17) are exactly doable in terms of gamma functions. In the limit $z \rightarrow 0$ we get $g_{1}(0)=1$, $g_{2}(0)=2 Q(0)=\Delta$ and $I(z)=z^{2} /(2 \Delta)+O\left(z^{4}\right)$. Therefore, in this limit, Eq. 19] reduces to a Gaussian form with a variance

$$
\left\langle X_{t}^{2}\right\rangle_{c}=\frac{\Delta \sigma_{t}}{\rho},
$$

which is the so-called Percus relation [12, 22]. The corrections to this result can obtained following a similar proceedure explained above [between Eqs. (20) and [21)]. The Gaussian form is expected to hold near the central region $\left|X_{t}\right| \lesssim O\left(\sqrt{\sigma_{t} / \rho}\right)$. However, away from this central region, the Gaussian approximation breaks down and one needs the complete form given by Eq. [19].

For a Gaussian propagator, we explicitly get

$$
Q(z)=\frac{e^{-z^{2} / 2}}{\sqrt{2 \pi}}+\frac{z}{2} \operatorname{erf}(z / \sqrt{2}), \text { and } A_{1}(z)=\frac{1}{2} \operatorname{erfc}(z / \sqrt{2}) .
$$

Using these expressions, in Fig. 2, we plot the (numerically normalized) large deviation form given by Eq. (2), the complete form given by Eq. (19) and its Gaussian approximation, and compare them with numerical simulation results. We note 


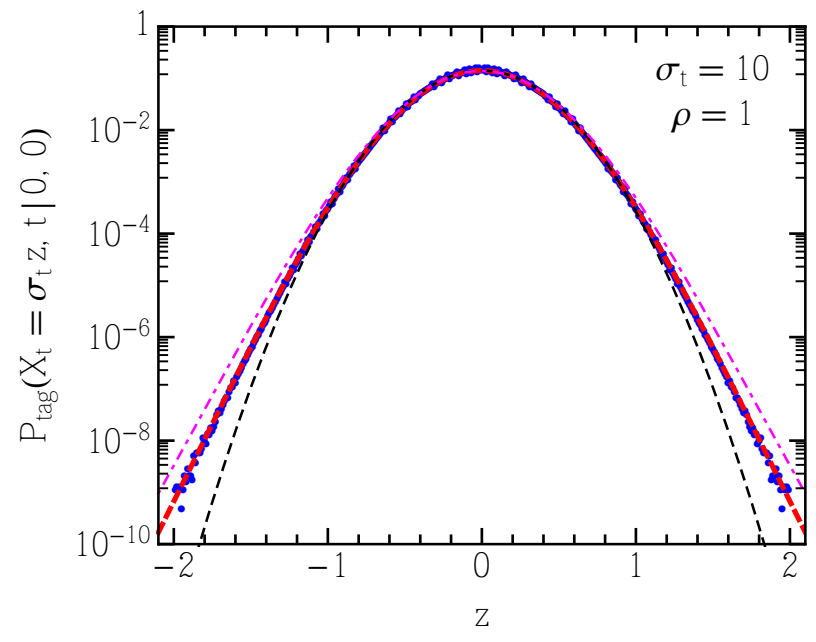

FIG. 2: (Color online) The (blue) points represent the simulation results for the PDF of the displacement of the tagged middle particle in a gas of $2 N+1$ particles initially distributed uniformly in a box between $[-N, N]$ with $N=1000$. Using the mapping to the non-interacting picture, each particle is evolved independently according to the Gaussian propagator with $\sigma_{t}=10$ and the difference in positions of the middle particles at the initial and the final times respectively is the displacement of the tagged middle particle in the interacting particle system. The PDF is computed using $32 \times 10^{9}$ realizations. The (red) thick dashed line corresponds to the analytic result in Eq. 19, while the (magenta) dot-dashed line plots the (numerically normalized) large deviation form given by Eq. 22. The (black) dashed line is Gaussian distribution with the variance given by Eq. 24a.

that the large deviation form of the PDF, given by Eq. (2), really implies the mathematical equality

$$
I(x)=-\lim _{\sigma_{t} \rightarrow \infty} \frac{1}{\rho \sigma_{t}} \ln P_{\mathrm{tag}}\left(X_{t}=x \sigma_{t}\right)
$$

However, to achieve the required large time limit for comparison with real data is often difficult, and it is necessary to include the sub-leading correction. Indeed, Eq. (19), which includes the correction term, agrees extremely well with the numerical simulation results. We note that, for diffusive systems, our result can be recovered by taking appropriate limits of the corresponding expressions in [16].

Now, we look at the cumulant generating function of the tagged particle displacement $X_{t}$, defined through

$$
Z(\lambda)=\left\langle e^{\lambda \rho X_{t}}\right\rangle=e^{\rho \sigma_{t} \mu(\lambda)}
$$

Using the large deviation form of $P_{\text {tag }}\left(X_{t}, t \mid 0,0\right)$ given by Eq. (2), and then evaluating the integral over $z$ using the saddle point approximation, we have $\mu(\lambda)=\lambda z^{*}-I\left(z^{*}\right)$ where $z^{*}$ is implicitly given by the equation $\lambda=I^{\prime}\left(z^{*}\right)$. Using the expression of $I(z)$ obtained above in terms of $\theta^{*}$ with the substitution $\theta^{*}=i B$, we can express $\mu(\lambda)$ in the parametric form
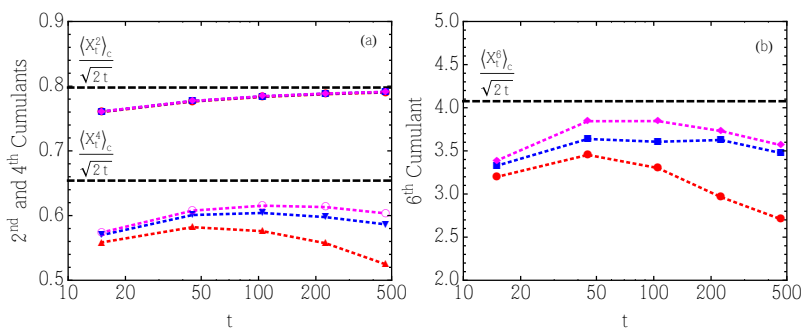

FIG. 3: (Color online) Points connected by dotted lines are the simulation results for (a) $2^{\text {nd }}, 4^{\text {th }}$ and (b) $6^{\text {th }}$ cumulants (scaled) of the displacement of the tagged middle particle in a gas of $2 N+1$ hard point diffusing particles $(D=1)$, initially distributed uniformly in a box between $[-N, N]$. The data is for system sizes $N=250$ (red, lowest curve), 500 (blue, middle curve) and 750 (magenta, upper curve). All cumulants are seen to approach our theoretical predictions (black dashed lines) with increasing system size.

$$
\begin{aligned}
& \mu(\lambda)=\left[\lambda+\frac{1-e^{B}}{1+e^{B}}\right] z \\
& \lambda=\left(1-e^{-B}\right)\left[1+\left(e^{B}-1\right) A_{1}(z)\right], \\
& e^{2 B}=\frac{2 Q(z)+z}{2 Q(z)-z}
\end{aligned}
$$

For the case of the Gaussian propagator with a variance $\sigma_{t}^{2}$, the first three even cumulants can be obtained as

$$
\begin{aligned}
\left\langle X_{t}^{2}\right\rangle_{c} & =\frac{\sqrt{2}}{\rho \sqrt{\pi}} \sigma_{t}, \\
\left\langle X_{t}^{4}\right\rangle_{c} & =\frac{3 \sqrt{2}(4-\pi)}{(\rho \sqrt{\pi})^{3}} \sigma_{t}, \\
\left\langle X_{t}^{6}\right\rangle_{c} & =\frac{15 \sqrt{2}\left(68-30 \pi+3 \pi^{2}\right)}{(\rho \sqrt{\pi})^{5}} \sigma_{t} .
\end{aligned}
$$

Figure 3 compares the above analytic expressions with the simulation results, for the case where individual particle motion is diffusive. Note that at large times, finite size effects kick in and the curves start deviating from the expected infinite size behavior. The higher cumulants sense the boundary effects at earlier times than the lower ones.

In conclusion, we have explicitly computed exactly the large time asymptotic form of the probability distribution of a tagged particle in a single-file system and shown that this is universal. This unifies the treatment of single-file motion of particles with hardcore interactions, within a general framework, as has also been attempted in some earlier work [20, 22]. For the case of Brownian particles, our results have been verified using macroscopic fluctuation theory [28]. However our microscopic approach is more intuitive from a physical point of view, it is more general, and directly gives the large deviation function as well as the important corrections often required for comparison with real data. The methods of the Letter can be extended to more general initial conditions. 
We thank K. Mallick, T. Sadhu, B. Derrida, and A. Roy for useful discussions. SS and AD acknowledge the hospitality of the GGI, Florence during the workshop "Advances in Nonequilbrium Statistical Mechanics (2014)" where part of this work was carried out. SS acknowledges the support of the Indo-French Centre for the Promotion of Advanced Research (IFCPAR/CEFIPRA) under Project 4604-3. AD thanks DST for support through the Swarnajayanti fellowship.

[1] A. L. Hodgkin and R. D. Keynes, J. Physiol. 128, 61 (1955).

[2] D. W. Jepsen, J. Math. Phys. 6, 405 (1965).

[3] T.E. Harris, J. Appl. Probab. 2,323 (1965).

[4] K. Hahn, J. Kärger, and V. Kukla, Phys. Rev. Lett. 76, 2762 (1996).

[5] V. Kulka et al., Science 272, 702 (1996).

[6] H. Wei, C. Bechinger, and P. Leiderer, Science 287, 625 (2000).

[7] C. Lutz, M. Kollmann and C. Bechinger, Phys. Rev. Lett. 93, 026001 (2004).

[8] B. Lin, M. Meron, B. Cui, S. A. Rice, and H. Diamant, Phys. Rev. Lett. 94, 216001 (2005).

[9] A. Das et al., ACS nano, 4, 1687 (2010).

[10] J. L. Lebowitz and J. K. Percus, Phys. Rev. 155, 122 (1967).

[11] J. L. Lebowitz and J. Sykes, J. Stat. Phys. 6, 157 (1972).
[12] J. K. Percus, Phys. Rev. A 9, 557 (1974).

[13] H.van Beijeren, K.W. Kehr, and R. Kutner, Phys. Rev. B 28, 5711 (1983).

[14] R. Arratia, Ann. Probab. 11, 362 (1983).

[15] S. Alexander and P. Pincus, Phys. Rev. B 18, 2011 (1978).

[16] C. Rödenbeck, J. Kärger, and K. Hahn, Phys. Rev. E 57, 4382 (1998).

[17] S. N. Majumdar and M. Barma, Phys. Rev. B 44, 5306 (1991).

[18] L. Lizana and T. Ambjörnsson, , Phys. Rev. Lett 100, 200601 (2008); Phys. Rev. E 80, 051103 (2009).

[19] E. Barkai and R. Silbey, Phys. Rev. Lett. 102, 050602 (2009).

[20] M. Kollmann, Phys. Rev. Lett. 90, 180602 (2003).

[21] S. Gupta, S. N. Majumdar, C. Godrèche and M. Barma, Phys. Rev. E 76, 021112 (2007).

[22] E. Barkai and R. Silbey, Phys. Rev. E 81, 041129 (2010).

[23] A. Roy, O. Narayan, A. Dhar and S. Sabhapandit, J. Stat. Phys. 150, 851 (2013).

[24] A. Roy, A. Dhar, O. Narayan and S. Sabhapandit, arXiv:1405.5718 (2014).

[25] S. Sabhapandit, J. Stat. Mech. L05002 (2007).

[26] P. Illien et el., Phys. Rev. Lett. 111, 038102 (2013).

[27] O. Bénichou et al., Phys. Rev. Lett. 111, 260601 (2013).

[28] P. L. Krapivsky, K. Mallick, and T. Sadhu, Phys. Rev. Lett. 113, 078101 (2014).

[29] G. Jona-Lasinio, Prog. Theo. Phys. Supp. 184, 262 (2010); J. Stat. Mech. P02004 (2014).

[30] Supplementary material.

\title{
Supplementary material for "Universal Large Deviations for the Tagged Particle in Single File Motion”
}

\author{
EVALUATION OF $F_{1 N}(x, y, t)$
}

Let $F_{1 N}(x, y, t)$ be the probability that there are an equal number of particles to the left and right of $x$ and $y$ at $t=0$ and $t$ respectively. In this case, one selected particle out of $2 N+1$ particles, goes from $x$ to $y$ in time $t$. The remaining $2 N$ particles are independent of each other and the selected particle. Let $p_{-+}(x, y, t)$ be the probability that one of these particles is to the left of $x$ at $t=0$ and to the right of $y$ at time $t$. Let $p_{+-}, p_{--}$and $p_{++}$be similarly defined.

Let $n_{1}$ be the number of particles that go from the left of $x$ to the left of $y, n_{2}$ be the number of particles going from left to the right, $n_{3}$ be the number of particles going from the right to the left, and $n_{4}$ be the number of particles going from the right to the right. Clearly $n_{1}+n_{2}+n_{3}+n_{4}=2 N$. Moreover $n_{1}+n_{2}=n_{3}+n_{4}$ and $n_{1}+n_{3}=n_{2}+n_{4}$, as there are equal number of particles on the two sides of the tagged particle at both the initial and final times. These equalities imply $n_{1}=n_{4}$ and $n_{2}=n_{3}$.

The number of ways of choosing the set $\left\{n_{1}, n_{2}, n_{3}, n_{4}\right\}$ is given by the multinomial coefficient

$$
\frac{(2 N) !}{n_{1} ! n_{2} ! n_{3} ! n_{4} !}
$$

and each possibility occurs with probability

$$
p_{--}^{n_{1}} p_{-+}^{n_{2}} p_{+-}^{n_{3}} p_{++}^{n_{4}} .
$$


Hence, summing over all possible values of $\left\{n_{1}, n_{2}, n_{3}, n_{4}\right\}$ we get

$$
F_{1 N}=\sum_{n_{1}+n_{2}+n_{3}+n_{4}=2 N} \frac{(2 N) !}{n_{1} ! n_{2} ! n_{3} ! n_{4} !} p_{--}^{n_{1}} p_{-+}^{n_{2}} p_{+-}^{n_{3}} p_{++}^{n_{4}} \delta_{n_{1}, n_{4}} \delta_{n_{2}, n_{3}}
$$

Now, after using the integral representation of the Kronecker delta,

$$
\delta_{m, n}=\frac{1}{2 \pi} \int_{-\pi}^{\pi} e^{i(m-n) \theta} d \theta
$$

in the above equation, it immediately follows that

$$
\begin{gathered}
F_{1 N}(x, y, t)=\int_{-\pi}^{\pi} \frac{d \phi}{2 \pi} \int_{-\pi}^{\pi} \frac{d \theta}{2 \pi}\left[p_{++}(x, y, t) e^{i \phi}+p_{--}(x, y, t) e^{-i \phi}+p_{+-}(x, y, t) e^{i \theta}+p_{-+}(x, y, t) e^{-i \theta}\right]^{2 N} \\
=\int_{-\pi}^{\pi} \frac{d \phi}{2 \pi} \int_{-\pi}^{\pi} \frac{d \theta}{2 \pi}\left[1-(1-\cos \phi)\left(p_{++}+p_{--}\right)+i \sin \phi\left(p_{++}-p_{--}\right)\right. \\
\left.-(1-\cos \theta)\left(p_{+-}+p_{-+}\right)+i \sin \theta\left(p_{+-}-p_{-+}\right)\right]^{2 N} \\
=\int_{-\pi / 2}^{\pi / 2} \frac{d \phi}{\pi} \int_{-\pi}^{\pi} \frac{d \phi}{2 \pi}\left[1-(1-\cos \phi)\left(p_{++}+p_{--}\right)+i \sin \phi\left(p_{++}-p_{--}\right)\right. \\
\left.-(1-\cos \theta)\left(p_{+-}+p_{-+}\right)+i \sin \theta\left(p_{+-}-p_{-+}\right)\right]^{2 N} .
\end{gathered}
$$

In the last step the range of the $\phi$ integral has been broken into two parts, and since $2 N$ is even, each of these contributes equally.

\section{EVALUATION OF $F_{2 N}(x, y, \tilde{x}, \tilde{y}, t)$}

Let $F_{2 N}(x, y, \tilde{x}, \tilde{y}, t)$ be the probability that there are an equal number of particles on both sides of $x$ and $y$ at $t=0$ and $t$ respectively, given that there is one particle at $(\tilde{x}, 0)$ and another one at $(\tilde{y}, t)$. The particle from $\tilde{x}$ goes to $y$ and the particle from $x$ goes to $\tilde{y}$ in time $t$. To compute $F_{2 N}$, one has to keep track of both these particles. There arises four situations (a) $\tilde{x}<x$ and $\tilde{y}<y$, (b) $\tilde{x}>x$ and $\tilde{y}>y$, (c) $\tilde{x}<x$ and $\tilde{y}>y$, and (d) $\tilde{x}>x$ and $\tilde{y}<y$.

Let there be $n_{1}$ particles going from the left of $x$ to the left of $y, n_{2}$ particles from the left to the right, $n_{3}$ particles from the right to the left, and $n_{4}$ particles from the right to the right. Since two of the particles are considered separately, the rest can be chosen in $(2 N-1) ! /\left(n_{1} ! n_{2} ! n_{3} ! n_{4} !\right)$ different ways and $n_{1}+n_{2}+n_{3}+n_{4}=2 N-1$.

Now, in the situation (a) we have, $n_{1}+n_{2}+1=n_{3}+n_{4}$ and $n_{1}+n_{3}+1=n_{2}+n_{4}$. These conditions are equivalent to $n_{2}=n_{4}$ and $n_{1}=n_{4}-1$. Similarly, the conditions for the other three situations can be worked out, and this gives (b) $n_{1}=n_{4}$ and $n_{2}=n_{3}-1$, (c) $n_{2}=n_{3}$ and $n_{1}=n_{4}+1$, and (d) $n_{1}=n_{4}$ and $n_{2}=n_{3}+1$, respectively.

Now following the procedure used to evaluate $F_{1 N}$, it is easily found that

$$
\begin{aligned}
F_{2 N}(x, y, \tilde{x}, \tilde{y}, t) & =\int_{-\pi}^{\pi} \frac{d \phi}{2 \pi} \int_{-\pi}^{\pi} \frac{d \theta}{2 \pi}\left[p_{++} e^{i \phi}+p_{--} e^{-i \phi}+p_{+-} e^{i \theta}+p_{-+} e^{-i \theta}\right]^{2 N-1} \psi(\theta, \phi), \\
& =\int_{-\pi / 2}^{\pi / 2} \frac{d \phi}{\pi} \int_{-\pi}^{\pi} \frac{d \theta}{2 \pi}\left[p_{++} e^{i \phi}+p_{--} e^{-i \phi}+p_{+-} e^{i \theta}+p_{-+} e^{-i \theta}\right]^{2 N-1} \psi(\theta, \phi),
\end{aligned}
$$

where the extra phase factor $\psi(\theta, \phi)$ originates from addend \pm 1 that appear in the relations among $n_{i}$ 's above, and $\psi(\theta, \phi)=$ $e^{-i \phi}, e^{i \phi}, e^{-i \theta}$, and $e^{i \theta}$ for the situations (a) $\tilde{x}<x$ and $\tilde{y}<y$, (b) $\tilde{x}>x$ and $\tilde{y}>y$, (c) $\tilde{x}<x$ and $\tilde{y}>y$, and (d) $\tilde{x}>x$ and $\tilde{y}<y$ respectively. To arrive at the last line, we have broken the integral over $\phi$ into two parts, and used the fact that $2 N-1$ is odd, and phase factors yield a extra factor of $(-1)$ when the phases are shifted by $\pi$. 